\title{
Carrier-Induced Magnetic Circular Dichloism in the Magnetoresistive Pyrochlore $\mathrm{Tl}_{2} \mathrm{Mn}_{2} \mathrm{O}_{7}$.
}

\author{
Hidekazu Okamura*, Toshihisa Koretsune, Shin-ichi Kimura ${ }^{1}$, Takao Nanba, \\ Hideto ImaI ${ }^{2}$, Yuichi Shimakawa ${ }^{2 \dagger}$ and Yoshimi KubO ${ }^{2}$ \\ Graduate School of Science and Technology, Kobe University, Kobe 657-8501 \\ ${ }^{1}$ UVSOR Facility, Institute for Molecular Science, Okazaki 444-8585 \\ 2 Fundamental Research Laboratories, NEC Corporation, Tsukuba 305-8501.
}

(Received January 29, 2019)

\begin{abstract}
Infrared magnetic circular dichloism (MCD), or equivalently magneto-optical Kerr effect, has been measured on the $\mathrm{Tl}_{2} \mathrm{Mn}_{2} \mathrm{O}_{7}$ pyrochlore, which is well known for exhibiting a large magnetoresistance around the Curie temperature $T_{C} \sim 120 \mathrm{~K}$. A circularly polarized, infrared synchrotron radiation is used as the light source. A pronounced MCD signal is observed exactly at the plasma edge of the reflectivity near and below $T_{C}$. However, contrary to the conventional behavior of MCD for ferromagnets, the observed MCD of $\mathrm{Tl}_{2} \mathrm{Mn}_{2} \mathrm{O}_{7}$ grows with the applied magnetic field, and not scaled with the internal magnetization. It is shown that these results can be basically understood in terms of a classical magnetoplasma resonance. The absence of a magnetization-scaled MCD indicates a weak spin-orbit coupling of the carriers in $\mathrm{Tl}_{2} \mathrm{Mn}_{2} \mathrm{O}_{7}$. We discuss the present results in terms of the microscopic electronic structures of $\mathrm{Tl}_{2} \mathrm{Mn}_{2} \mathrm{O}_{7}$.
\end{abstract}

KEYWORDS: $\mathrm{Tl}_{2} \mathrm{Mn}_{2} \mathrm{O}_{7}$, infrared magnetic circular dichloism, magnetoplasma, colossal magnetoresistance.

\section{Introduction}

Physics of colossal magneto-resistance (CMR) phenomena has attracted much interest recently. The most famous examples are probably the ferromagnetic perovskite manganites such as $\mathrm{La}_{1-x} \mathrm{Sr}_{x} \mathrm{MnO}_{3} \cdot{ }^{1}$ In these compounds, the so-called "double exchange" interaction and the strong Jahn-Teller effect due to $\mathrm{Mn}^{3+}$ are responsible for the CMR and other interesting properties near the Curie temperature $\left(T_{C}\right){ }^{2,3}$ Another CMR compound that is equally interesting is the $\mathrm{Tl}_{2} \mathrm{Mn}_{2} \mathrm{O}_{7}$ pyrochlore. ${ }^{4-9}$ This compound is also a ferromagnet, and its resistivity $(\rho)$ drops rapidly upon cooling below $T_{C} \sim$ $120 \mathrm{~K}$. Near and above $T_{C}$, an external magnetic field $(B)$ of $7 \mathrm{~T}$ reduces $\rho$ by a factor of $\sim 10$. Although these features appear similar to those for the perovskites, it has been shown that the underlying mechanism is very different: The spontaneous magnetization below $T_{C}$ is produced by the $\mathrm{Mn}^{4+}$ sublattice through superexchange interaction, independently from the conduction electrons. The conduction band has large $\mathrm{Tl} 6 s$ and $\mathrm{O} 2 p$ components. ${ }^{8,10,11}$ The CMR mainly results from changes in the conduction band when the applied $B$ field induces a magnetization and hence a band polarization. Such magneto-resistance mechanism has been considered ${ }^{12,13}$ to be analogous to that of europium chalcogenides such as $\mathrm{EuO}$.

Our previous magneto-optical study of $\mathrm{Tl}_{2} \mathrm{Mn}_{2} \mathrm{O}_{7}$ provided much information regarding its electronic structures. ${ }^{14}$ The optical reflectivity spectrum $R(\omega)$ at $295 \mathrm{~K}$ was similar to those for insulating oxides. Upon cooling below $T_{C}$, however, $R(\omega)$ showed marked increases in the far-infrared region, with a clear plasma edge un-

*E-mail: okamura@phys.sci.kobe-u.ac.jp

${ }^{\dagger}$ Present Address: Institute for Chemical Research, Kyoto University, Uji 611-0011. dergoing blue shifts with decreasing temperature $(T)$. These changes clearly indicated a crossover to metallic electronic structures below $T_{C} . R(\omega)$ also showed large increases and plasma edge shifts when a strong magnetic field was applied near $T_{C}$, that were very similar to those caused by cooling. Based on the $R(\omega)$ data and the optical conductivity $\sigma(\omega)$ obtained from $R(\omega)$, we concluded that the CMR was caused by the appearance of a small conduction band, and that the $B$-induced changes in the electronic structure were very similar to those induced by cooling at $B=0$. It was also found that the effective carrier density showed a universal scaling with $M^{2}$, where $M$ is the internal magnetization, over wide ranges of $T$ and $B$.

In this work we have studied the magnetic circular dichloism (MCD) of $\mathrm{Tl}_{2} \mathrm{Mn}_{2} \mathrm{O}_{7}$ in order to obtain information about spin-dependent carrier dynamics below $T_{C}$. An MCD refers to a magnetically-induced difference between the optical responses to the incident light with right- and left-circular polarizations. In principle it can be a sensitive probe of spin-polarized electronic states in a magnetic material. Band calculations for ferromagnetic $\mathrm{Tl}_{2} \mathrm{Mn}_{2} \mathrm{O}_{7}$ have predicted that the conduction electrons are completely spin-polarized. ${ }^{8,10,11}$ The carrier dynamics of such spin-polarized material is very interesting from both physical and technological points of view. A strong MCD has been observed exactly at the plasma edge of $R(\omega)$ in the infrared region, showing that the MCD arises from a small density of free carriers. The appearance of the MCD is closely related to the applied $B$, rather than $M$. We show that the observed MCD spectra can be basically understood based on a classical magneto-plasma model. The results are analyzed in terms of the electronic structures of $\mathrm{Tl}_{2} \mathrm{Mn}_{2} \mathrm{O}_{7}$. 


\section{Experimental}

We used the same $\mathrm{Tl}_{2} \mathrm{Mn}_{2} \mathrm{O}_{7}$ sample, having $T_{C} \sim 120 \mathrm{~K}$, as that used in the previous report. ${ }^{14}$ The MCD experiments were done using a circularly polarized infrared synchrotron radiation as the light source, at the beam line BL6A1 of the UVSOR Facility, Institute for Molecular Science. ${ }^{15,16}$ Using synchrotron radiation source, one can obtain circularly polarized light over a wide range of photon energy, ${ }^{15-17}$ without additional optical elements such as a $\lambda / 4$ plate. The reflectivity spectra of the sample were measured under a near-normal incidence, with magnetic fields applied perpendicular to the sample surface. An MCD under this condition is often referred to as the magneto-optical Kerr effect. ${ }^{18}$ The incident light always had right circular polarization, and the magnetic field was applied either parallel $\left(\vec{B}_{+}\right)$or antiparallel $\left(\vec{B}_{-}\right)$to the incident light. In this work we define the MCD spectrum as

$$
\delta(\omega)=-\frac{R_{+}(\omega)-R_{-}(\omega)}{R_{\text {avg }}(\omega)},
$$

where $R_{ \pm}(\omega)$ are the $R(\omega)$ spectra measured under $\vec{B}_{ \pm}$ fields, respectively, and $R_{a v g}(\omega)$ is the average of $R_{ \pm}(\omega)$. Namely the MCD is the relative difference in $R(\omega)$ that results when the $\vec{B}$ direction is reversed for a fixed helicity of circularly polarized incident light. (This is equivalent to reversing the helicity of the circular polarization for a fixed $\vec{B}$ direction.) The MCD thus defined is directly related to the Kerr ellipticity, $\eta_{K}(\omega)$, as $\delta(\omega)=-4 \eta_{K}(\omega) .{ }^{18}$ The Kerr rotation angle $\theta_{K}(\omega)$ is given as

$$
\theta_{K}(\omega)=-\left[\theta_{+}(\omega)-\theta_{-}(\omega)\right] / 2 .
$$

Here $\theta_{ \pm}(\omega)$ are the phase shifts of the complex reflectivity under $\vec{B}_{ \pm}$fields, which can be obtained from the $R_{ \pm}(\omega)$ spectra using the Kramers-Kronig (K-K) relations. ${ }^{19}$ A superconducting magnet was used to apply the field, and a Fourier-transform interferometer was used to record the reflectivity spectra. The magneto-optical spectra were taken at photon energies below $1.1 \mathrm{eV}$, to which the zero-field spectra up to $35 \mathrm{eV}^{14}$ were smoothly connected.

\section{Results and Discussion}

Figure 1 shows the $R_{\text {avg }}(\omega)$ and the MCD spectra of $\mathrm{Tl}_{2} \mathrm{Mn}_{2} \mathrm{O}_{7}$ at $40 \mathrm{~K}$ for $B=2,4$, and $6 \mathrm{~T}$. $R_{\text {avg }}(\omega)$ at $40 \mathrm{~K}$ shows only minor dependence on $B,{ }^{14}$ and the $R_{\text {avg }}(\omega)$ spectra at 0 and $6 \mathrm{~T}$ in Fig. 1 are almost unchanged. The sharp minimum in $R_{\text {avg }}(\omega)$ near $0.17 \mathrm{eV}$ is the plasma edge, below which the reflectivity is high due to the Drude response of free carriers. The sharp structures in $R_{\text {avg }}(\omega)$ below $0.08 \mathrm{eV}$ are due to optical phonons. As shown in the bottom graph of Fig. 1, a clear MCD is observed around the plasma edge, which becomes stronger with increasing $B$. The sharp MCD peaks around $0.05 \mathrm{eV}$ probably result from the plasmon-LO phonon coupling, ${ }^{20}$ which will not be discussed in this work. The Kerr rotation $\theta_{K}(\omega)$ at $6 \mathrm{~T}$ is also shown in Fig. 1. It is seen that the maximum Kerr rotation of $\mathrm{Tl}_{2} \mathrm{Mn}_{2} \mathrm{O}_{7}$ is about 1 degree at $B=6 \mathrm{~T}$.
The temperature dependence of the observed MCD at $B=6 \mathrm{~T}$ is shown in Fig. 2, together with the $R_{\text {avg }}(\omega)$ spectra at 0 and $6 \mathrm{~T}$. The field-induced variations of $R_{\text {avg }}(\omega)$ at $125 \mathrm{~K}$, near $T_{C}$, are very large. This is due to the increase of free carriers caused by the band polarization induced by the applied magnetic field,${ }^{14}$ which is closely related with the CMR. Away from $T_{C}$, however, the field-induced changes in $R_{\text {avg }}(\omega)$ are very small. The observed MCD becomes stronger with decreasing $T$. In addition, the position of the MCD peak closely follows that of the plasma edge, as the latter undergoes blue shifts with decreasing $T$. This result strongly suggests that the observed $M C D$ is closely related with the free carriers in $\mathrm{Tl}_{2} \mathrm{Mn}_{2} \mathrm{O}_{7}$. Note that the field-induced changes of $R_{\text {avg }}(\omega)$ are largest near $T_{C}$, but the MCD becomes largest at lower temperatures.

Figure 3 plots the magnitude (peak height) of the $\mathrm{MCD}$ as functions of magnetic field and temperature, with the measured magnetization $(M)$ of the same sample. Note that $M$ is induced even well above $T_{C}$ under applied fields of 4 and 6 T. Figure 3 clearly shows that the variation of MCD does not closely follow that of $M$. This is most clearly seen at $40 \mathrm{~K}$ in Fig.3(a), where $M$ is almost saturated above $0.5 \mathrm{~T}$ although the MCD grows with increasing $B$ even above $4 \mathrm{~T}$. In addition, Fig. 3(b) shows that the MCD appears only below $160 \mathrm{~K}$, which is coincident with a rapid increase of $M$ with cooling. This result probably indicates that a well-defined conduction band can be established only below $160 \mathrm{~K}$, although an induced $M$ exists even at higher temperatures.

An MCD due to free carriers may arise through several mechanisms. In a ferromagnetic metal such as Gd, free carriers may lead to an MCD due to their skew scatterings caused by the internal $M$ and a spin-orbit coupling. ${ }^{21}$ Also for paramagnetic metals, a large MCD at $\omega_{p}$ has been attributed to an exchange-enhanced splitting of the plasma edge. ${ }^{22}$ In these cases, the MCD should be proportional to the spin polarization of the carriers. This is in contrast to the present results, where the observed MCD is not scaled with the internal $M$. Another possibility is a plasma edge-enhancement of $\mathrm{MCD},{ }^{23-25}$ where a strong $\mathrm{MCD}$ is caused when a steep plasma edge is present and well separated in energy from the interband transitions. Although the $R(\omega)$ spectra of $\mathrm{Tl}_{2} \mathrm{Mn}_{2} \mathrm{O}_{7}$ below $T_{C}$ may satisfy both of these conditions, ${ }^{14}$ this mechanism does not seem consistent with the $B$-dependence of the MCD for $\mathrm{Tl}_{2} \mathrm{Mn}_{2} \mathrm{O}_{7}$. Interaction of the plasma resonance with interband transitions involving magnetic states ${ }^{26}$ is also unlikely, since $\omega_{p}$ is far apart from the interband transitions, located above $2 \mathrm{eV} .{ }^{14}$ We show below that the classical magnetoplasma (MP) resonance, ${ }^{20,27}$ which is a coupled Drude-cyclotron response of free carriers under $B$ field, can basically account for the observed MCD. In this model an MCD appears at the plasma edge due to its splitting into $\omega_{p} \pm \omega_{c}$, where $\omega_{c}$ is the cyclotron energy.

We take the $z$ axis normal to the sample surface, and the polarization vectors lie in the $x y$ plane. Then in the classical MP model for isotropic band electrons, the com- 
ponents of the complex dielectric tensor are given $\operatorname{as}^{20,27}$

$$
\begin{aligned}
& \hat{\epsilon}_{x x}=1-\frac{\omega_{p}^{2}}{\omega} \frac{(\omega+i \gamma)}{(\omega+i \gamma)^{2}-\omega_{c}^{2}} \\
& \hat{\epsilon}_{x y}=-\hat{\epsilon}_{y x}=\frac{\omega_{p}^{2}}{\omega} \frac{i \omega_{c}}{(\omega+i \gamma)^{2}-\omega_{c}^{2}},
\end{aligned}
$$

where $\omega_{p}$ and $\omega_{c}$ are the plasma and cyclotron energies, respectively, and $\gamma$ is the damping. The complex refractive indeces for \pm field directions, $\hat{N}_{ \pm}$, are the two eigen values of the complex refractive index tensor. ${ }^{18}$ They satisfy $\hat{N}_{ \pm}^{2}=\hat{\epsilon}_{x x} \pm i \hat{\epsilon}_{x y}$. By definition, they also satisfy $\hat{N}_{ \pm}^{2}=\hat{\epsilon}_{ \pm}=\epsilon_{1 \pm}+i \epsilon_{2 \pm}$, where $\hat{\epsilon}_{ \pm}$are the complex dielectric functions of the MP for the \pm field directions, and $\epsilon_{1 \pm}$ and $\epsilon_{2 \pm}$ are their real and imaginary parts, respectively. Hence we have

$$
\hat{\epsilon}_{x x} \pm i \hat{\epsilon}_{x y}=\epsilon_{1 \pm}+i \epsilon_{2 \pm}
$$

Substituting (3) and (4) into (5), we obtain

$$
\begin{aligned}
& \epsilon_{1 \pm}=1-\frac{\omega_{p}^{2}}{\omega^{2}+\gamma^{2}} \mp \frac{\omega_{p}^{2} \omega_{c}\left(\omega^{2}-\gamma^{2}\right)}{\omega\left(\omega^{2}+\gamma^{2}\right)^{2}} \\
& \epsilon_{2 \pm}=\frac{\gamma \omega_{p}^{2}}{\omega\left(\omega^{2}+\gamma^{2}\right)} \pm \frac{2 \gamma \omega_{p}^{2} \omega_{c}}{\left(\omega^{2}+\gamma^{2}\right)^{2}},
\end{aligned}
$$

where we have used $\left(\omega_{c} / \omega_{p}\right) \ll 1$, valid for the present case. The total dielectric function is expressed as ${ }^{19}$

$$
\hat{\epsilon}_{ \pm}^{t}=\hat{\epsilon}_{ \pm}+\hat{\epsilon}^{i b}+\hat{\epsilon}^{p h},
$$

where $\hat{\epsilon}^{i b}$ arises from the higher-energy interband transitions, which partially screens the plasma oscillations, and $\hat{\epsilon}^{p h}$ arises from the optical phonons. $R_{ \pm}(\omega)$ are given as ${ }^{19}$

$$
R_{ \pm}(\omega)=\frac{\left(n_{ \pm}-1\right)^{2}+k_{ \pm}^{2}}{\left(n_{ \pm}+1\right)^{2}+k_{ \pm}^{2}}
$$

where $n_{ \pm}$and $k_{ \pm}$are real and imaginary refractive indices, respectively, obtained by solving $\epsilon_{1 \pm}^{t}=n_{ \pm}^{2}-k_{ \pm}^{2}$ and $\epsilon_{2 \pm}^{t}=2 n_{ \pm} k_{ \pm}$. Finally, the MCD in this model is obtained by substituting (9) into (1).

We simulate the observed MCD spectra using the above model as follows. First, we obtained the Drude parameters $\gamma$ and $\omega_{p}$ by fitting the observed $R_{\text {avg }}(\omega)$ based on the regular Drude reflectivity, i.e., that obtained by setting $\omega_{c}=0$ in (6)-(9). In doing so, we also used the classical Lorentz oscillator model ${ }^{19}$ for the phonon part $\epsilon^{p h}(\omega)$, and the actual $\epsilon^{i b}(\omega)$ obtained from the measured $R_{\text {avg }}(\omega)$ through the K-K relations. ${ }^{28}$ The observed $R_{\text {avg }}(\omega)$ spectra could be fitted well by this procedure, as shown in Fig. 4 (left axis). Then, we substituted the obtained $\gamma$ and $\omega_{p}$ into (6)-(9), and adjusted $\omega_{c}$ as a parameter so that the MCD calculated through (1) reproduces the observed MCD spectrum. The fitted value of $\omega_{c}$ was used to calculate the effective mass $m^{*}$, using the relation $\hbar \omega_{c}=e B_{i} / m^{*} c$. Here, we have assumed that the internal field $B_{i}$ acting on the electrons is equal to the applied field. ${ }^{29}$ Figure 4 shows the simulated reflectivity and MCD spectra at $6 \mathrm{~T}$, with the used parameters shown in the caption, and Fig. 5 plots the obtained $m^{*}$ values at different values of $B$ and $T$. The simulated spectra in Fig. 4 have well reproduced the observed MCD spectra in Fig. 2, with the obtained $m^{*} / m_{0}$ values of $\sim$
0.8 at $4 \mathrm{~T}$, and $\sim 0.6$ at $6 \mathrm{~T}$. These values are very reasonable, since they are close to those predicted by the band calculations for ferromagnetic $\mathrm{Tl}_{2} \mathrm{Mn}_{2} \mathrm{O}_{7}, 8,10,11$ and also to those estimated previously from the effective carrier density. ${ }^{14}$ Since the parameters $\gamma$ and $\omega_{p}$ have been chosen to reproduce $R_{a v g}(\omega)$, the only free parameter in simulating the MCD is $m^{*}$. Considering this fact and the simpleness of the model, the agreement between the data and the simulation is remarkable. The decrease in $m^{*}$ from $B=4 \mathrm{~T}$ to $6 \mathrm{~T}$ is likely to result from changes in the band structure caused by the increase of $M$. In contrast, $m^{*}$ does not show significant temperature dependence both at 4 and $6 \mathrm{~T}$.

Regarding the field dependence of the MCD, there seems to be a threshold for the onset of MCD, as seen in Fig. 3(a): the MCD grows with $B$ for 4 and $6 \mathrm{~T}$, but is almost absent at $2 \mathrm{~T}$. This is unexpected from the simple MP model, which predicts that the MCD at $2 \mathrm{~T}$ is about half that at $4 \mathrm{~T}$. (Note that a well-defined conduction band exists for $T \ll T_{C}$ even at zero field.) The reason for this threshold behavior is unclear at the present, and it is likely to result from complications not included in the simple M-P model. For example, the electrons experience scattering due to disorder such as defects and impurities, in addition to electron-phonon interaction. The degree of disorder-related scattering may vary with the field strength, depending on the relative magnitude of the magnetic length (cyclotron radius) to the spatial size of the disorder potential. Such scattering may make the contribution of cyclotron motion to the MP resonance observed only above certain field strength.

Our previous work ${ }^{14}$ has shown that the variations of $R(\omega)$ and $\sigma(\omega)$ as functions of $B$ and $T$ closely follow the associated variations of the internal magnetization $M$. In fact, the effective carrier density evaluated from the measured spectra scaled with $M^{2}$ over wide ranges of $T$ and $B$. In addition, the band calculations have predicted that the conduction electrons in $\mathrm{Tl}_{2} \mathrm{Mn}_{2} \mathrm{O}_{7}$ are completely spin polarized. In view of these previous results, the absence of an $M$-scaled MCD for $\mathrm{Tl}_{2} \mathrm{Mn}_{2} \mathrm{O}_{7}$ is a rather unexpected result. Since the angular momentum carried by a circularly polarized photon cannot directly couple with the electron spin and hence with $M$, the carriers must have a sufficient spin-orbit $(s-o)$ coupling to lead to an MCD. Therefore the present result strongly suggests a weak $s$-o coupling for the conduction electrons in $\mathrm{Tl}_{2} \mathrm{Mn}_{2} \mathrm{O}_{7}$. A weak s-o coupling of carries in $\mathrm{Tl}_{2} \mathrm{Mn}_{2} \mathrm{O}_{7}$ has also been indicated by the observation of a very weak anomalous Hall effect even well below $T_{C}{ }^{9}$ According to the band calculations, the density of states for the conduction band below the Fermi level in ferromagnetic $\mathrm{Tl}_{2} \mathrm{Mn}_{2} \mathrm{O}_{7}$ are mainly derived from the $\mathrm{Tl} 6 \mathrm{~s}$, O $2 p$, and Mn $3 d$ states about 1:1:1 ratio. Among these, the orbital angular momentum for $3 d$ electron is usually quenched, so that the O $2 p$ component may be the only source of $s-o$ coupling for the conduction electrons. This may account for the absence of an $M$-dependent MCD for $\mathrm{Tl}_{2} \mathrm{Mn}_{2} \mathrm{O}_{7}$. For a more quantitative account of the observed MCD, however, it is probably necessary to take into account the actual band structures, as well as their dependence on the magnetization. 
It is interesting to compare the present results of $\mathrm{Tl}_{2} \mathrm{Mn}_{2} \mathrm{O}_{7}$ with those of $\mathrm{EuB}_{6},{ }^{31-33}$ another ferromagnet with $T_{C}=16 \mathrm{~K}$ which shows large magneto-optical effects similar to those for $\mathrm{Tl}_{2} \mathrm{Mn}_{2} \mathrm{O}_{7}$. Near $T_{C}, \mathrm{EuB}_{6}$ shows very large shifts of the plasma edge in $R(\omega)$ with $T$ and $B .{ }^{31,32}$ The Drude weight (effective carrier density) scales with $M^{2}$ over wide ranges of $T$ and $B .{ }^{32} \mathrm{~A}$ pronounced MCD appears at the plasma edge of $R(\omega)$, with a Kerr rotation angle as large as 8 degrees. ${ }^{33}$ Although these results are qualitatively similar to those found for $\mathrm{Tl}_{2} \mathrm{Mn}_{2} \mathrm{O}_{7}$, there is one marked difference concerning the plasma edge MCD: In addition to the much larger magnitude, the $\mathrm{MCD}$ for $\mathrm{EuB}_{6}$ follows the variation of $M$ more closely than that for $\mathrm{Tl}_{2} \mathrm{Mn}_{2} \mathrm{O}_{7} \cdot{ }^{33}$ This is because the plasma edge MCD for $\mathrm{EuB}_{6}$ is due to the spin polarization of the localized Eu $4 f$ electrons under $B$ fields, aided by the coupling between the Drude dynamics and the nearby interband transitions involving $\mathrm{Eu} 4 f$ states. $^{33}$ This result is reasonable, since the $\mathrm{Eu} 4 f$ electrons are directly responsible for the $M$. In contrast, in the case of $\mathrm{Tl}_{2} \mathrm{Mn}_{2} \mathrm{O}_{7}$ the source of MCD is the free carriers themselves, which have only small $s$-o coupling resulting in the absence of $M$-scaled MCD.

\section{Conclusion}

We have studied the infrared MCD (magnetooptical Kerr effect) of the magnetoresistive pyrochlore $\mathrm{Tl}_{2} \mathrm{Mn}_{2} \mathrm{O}_{7}$, using synchrotron radiation source. A pronounced MCD signal has been observed exactly at the plasma edge of the reflectivity. The observed MCD grows with the applied external magnetic field, and it is not scaled with the internal magnetization. The MCD has been successfully analyzed in terms of the classical magnetoplasma resonance model. The absence of strongly $M$-dependent MCD in $\mathrm{Tl}_{2} \mathrm{Mn}_{2} \mathrm{O}_{7}$ indicates a weak spinorbit coupling of the conduction electrons in $\mathrm{Tl}_{2} \mathrm{Mn}_{2} \mathrm{O}_{7}$.

\section{Acknowledgements}

This work was performed as a joint studies program of the Institute for Molecular Science (2001). This work was partly supported by Grants-In-Aid from the MEXT.

1) See, for example, Y. Tokura, A. Urushibara, Y. Moritomo, T. Arima, A. Asamitsu, G. Kido and N. Furukawa: J. Phys. Soc. Jpn. 63 (1994) 3931.

2) C. Zener: Phys. Rev. 82 (1951) 403.

3) A.J. Millis, P.B. Littlewood and B.I. Shraiman: Phys. Rev. Lett. 74 (1995) 5144.

4) Y. Shimakawa, Y. Kubo and T. Manako: Nature 379 (1996) 53 ,

5) M.A. Subramanian, B.H. Toby, A.P. Ramirez, W.J. Marshall, A.W. Sleight and G.H. Kwei: Science 273 (1996) 81.

6) S.-W. Cheong, H.Y. Hwang, B. Batlogg and L.W. Rupp, Jr.: Solid State Commun. 98 (1996) 163.

7) Y. Shimakawa, Y. Kubo, T. Manako, Y.V. Sushko, D.N. Argyriou and J.D. Jorgensen: Phys. Rev. B 55 (1997) 6399.

8) Y. Shimakawa, Y. Kubo, N. Hamada, J.D. Jorgensen, Z. Hu, S. Short, M. Nohara and H. Takagi: Phys. Rev. B 59 (1999) 1249.

9) H. Imai, Y. Shimakawa, Yu.V. Sushko and Y. Kubo, Phys. Rev. B 62 (2000) 12190.

10) D.J. Singh: Phys. Rev. B 55 (1997) 313.

11) S.K. Mishra and S. Satphathy, Phys. Rev. B 58 (1998) 7585.
12) Y. Shimakawa and Y. Kubo: Mater. Sci. Eng. B 63 (1999) 44.

13) M. Kataoka: Phys. Rev. B 63 (2001) 134435.

14) H. Okamura, T. Koretsune, M. Matsunami, S. Kimura, T. Nanba, H. Imai, Y. Shimakawa, Y. Kubo: Phys. Rev. B 64 (2001) 180409.

15) S. Kimura: Jpn. J. Appl. Phys. 38 Suppl. 38-1 (1999) 392.

16) S. Kimura, M. Okuno, H. Iwata, H. Kitazawa, G. Kido, F. Ishiyama, O. Sakai: J. Phys. Soc. Jpn. 71 (2002) 2200.

17) Actually, the incident light used was not completely circularly polarized, but elliptically polarized. However, the degree of circular polarization was estimated to be greater than $90 \% .{ }^{15}$ Hence the uncertainty in the measured MCD caused by the incomplete circular polarization is less than $10 \%$.

18) S. Sugano and K. Kojima (Eds.), Magneto-optics (Springer, Berlin, 2000).

19) M. Dressel and G. Gruner: Electrodynamics of Solids (Cambridge University Press, Cambridge, 2002).

20) R.W. Stimets and B. Lax: Phys. Rev. B 1 (1970) 4720.

21) J.L. Erskine and E.A. Stern: Phys. Rev. B 8 (1973) 1239.

22) W. Reim, O.E. Husser, J. Schoenes, E. Kaldis, P. Wachter and K. Seiler: J. Appl. Phys. 55 (1984) 2155.

23) H. Feil and C. Haas: Phys. Rev. Lett. 58 (1987) 65.

24) J. Schoenes and W. Reim: Phys. Rev. Lett. 60 (1988) 1988.

25) H. Feil and C. Haas: Phy. Rev. Lett. 60 (1988) 1989.

26) F. Salghetti-Drioli, P. Wachter and L. Degirgi: Solid State Commun. 109 (1999) 773.

27) B. Lax and G.B. Wright: Phys. Rev. Lett. 4 (1960) 16.

28) In the measured spectra of $\mathrm{Tl}_{2} \mathrm{Mn}_{2} \mathrm{O}_{7}$, the interband transition peaks were well separated in energy from the DrudeLorentz components. ${ }^{14}$ Hence $\epsilon^{i b}(\omega)$ could be easily isolated from the measured total $\epsilon^{t}(\omega)$. The factor 1 in (5) was dropped since it should be included in $\epsilon^{i b}(\omega) .{ }^{19}$

29 ) In the presence of a magnetiztion $\vec{M}$, the internal field $\vec{B}_{i}$ may differ from the applied field $\vec{B}_{0}$, since $\vec{M}$ produces an additional field $\vec{B}_{m}$, where $\vec{B}_{i}=\vec{B}_{0}+\vec{B}_{m}$. The detail of $\vec{B}_{m}$ depends on the sample shape and $\vec{M}$. If there is a strong $\vec{B}_{m}$ in the $\mathrm{Tl}_{2} \mathrm{Mn}_{2} \mathrm{O}_{7}$ sample, this should appear, well below $T_{C}$, as a very rapid increase of the (ordinary) Hall voltage for $B_{0}<0.5 \mathrm{~T}$ [see Fig. 3(a)], and it should scale with $M$ rather than with $B_{0}$. However, no such feature was observed in the experiments ${ }^{9}$ for a sample having similar shape as that used in the present work. In addition, for a wide, plate-shaped sample with $\vec{M}$ and $\vec{B}_{0}$ perpendicular to the sample surface, it is well known that $\vec{B}_{i}=\vec{B}_{0}$. Our sample was a flat plate of $\sim 5 \times 5 \times 1 \mathrm{~mm}^{3}$. From these facts, we believe that $\vec{B}_{i} \simeq \vec{B}_{0}$ in our experiments.

30) The $\omega_{p}$ values obtained by fitting are much larger than the observed plasma edge positions in $R(\omega)$, since the fitted values are "bare" plasma energies while those observed in $R(\omega)$ have been reduced due to the screening by $\epsilon^{i b}$.

31) L. Degiorgi, E. Felder, H.R. Ott, J.L. Sarrao and Z. Fisk: Phys. Rev. Lett. 79 (1997) 5134.

32) S. Broderick, B. Ruzicka, L. Degiorgi, H.R. Ott, J.L. Sarrao and Z. FIsk: Phys. Rev. B 65 (2002) 121102(R).

33) S. Broderick, L. Degiorgi, H.R. Ott, J.J. Sarrao and Z. Fisk: Eur. Phys. J. B 33 (2003) 47. 
Fig. 1. Top graph: the reflectivity $(R)$ spectrum of $\mathrm{Tl}_{2} \mathrm{Mn}_{2} \mathrm{O}_{7}$ at $T=40 \mathrm{~K}$ and $B=0$ and $6 \mathrm{~T}$. Bottom panel: the MCD spectra, defined by (1), and the Kerr rotation $\theta_{K}$ measured at $T=40 \mathrm{~K}$ for $B=2,4$, and $6 \mathrm{~T}$.

Fig. 2. Unpolarized reflectivity spectra at magnetic fields $0 \mathrm{~T}$ (dotted curves) and $6 \mathrm{~T}$ (solid curves), and the MCD at $6 \mathrm{~T}$ (squares) of $\mathrm{Tl}_{2} \mathrm{Mn}_{2} \mathrm{O}_{7}$ measured at four temperatures. $\mathrm{MCD}$ signals below $0.06 \mathrm{eV}$ have been omitted for clarity.

Fig. 3. The magnitude of measured MCD (circles, squares, and triangles) plotted on the right axis as functions of (a) magnetic field and (b) temperature. The magnetization $(M)$ of the sample is also plotted on the left axis (solid, dotted, and dashed-dotted curves).
Fig. 4. Simulated reflectivity and MCD spectra at $6 \mathrm{~T}$ based on the MP model, as described in the text. The Drude parameters used are $\left(\omega_{p}, \gamma\right)=(0.24 \mathrm{eV}, 52 \mathrm{meV})$ for $140 \mathrm{~K},(0.325 \mathrm{eV}$, $39 \mathrm{meV})$ for $125 \mathrm{~K},(0.38 \mathrm{eV}, 30 \mathrm{meV})$ for $100 \mathrm{~K}$, and $(0.445 \mathrm{eV}$, $30 \mathrm{meV}$ ) for $40 \mathrm{~K}$. See also the note. ${ }^{30}$

Fig. 5. Effective masses in units of the rest electron mass $\left(\mathrm{m}^{*} / \mathrm{m}_{0}\right)$ obtained from the MCD data at magnetic fields $B=4$ and $6 \mathrm{~T}$ as a function of temperature. 

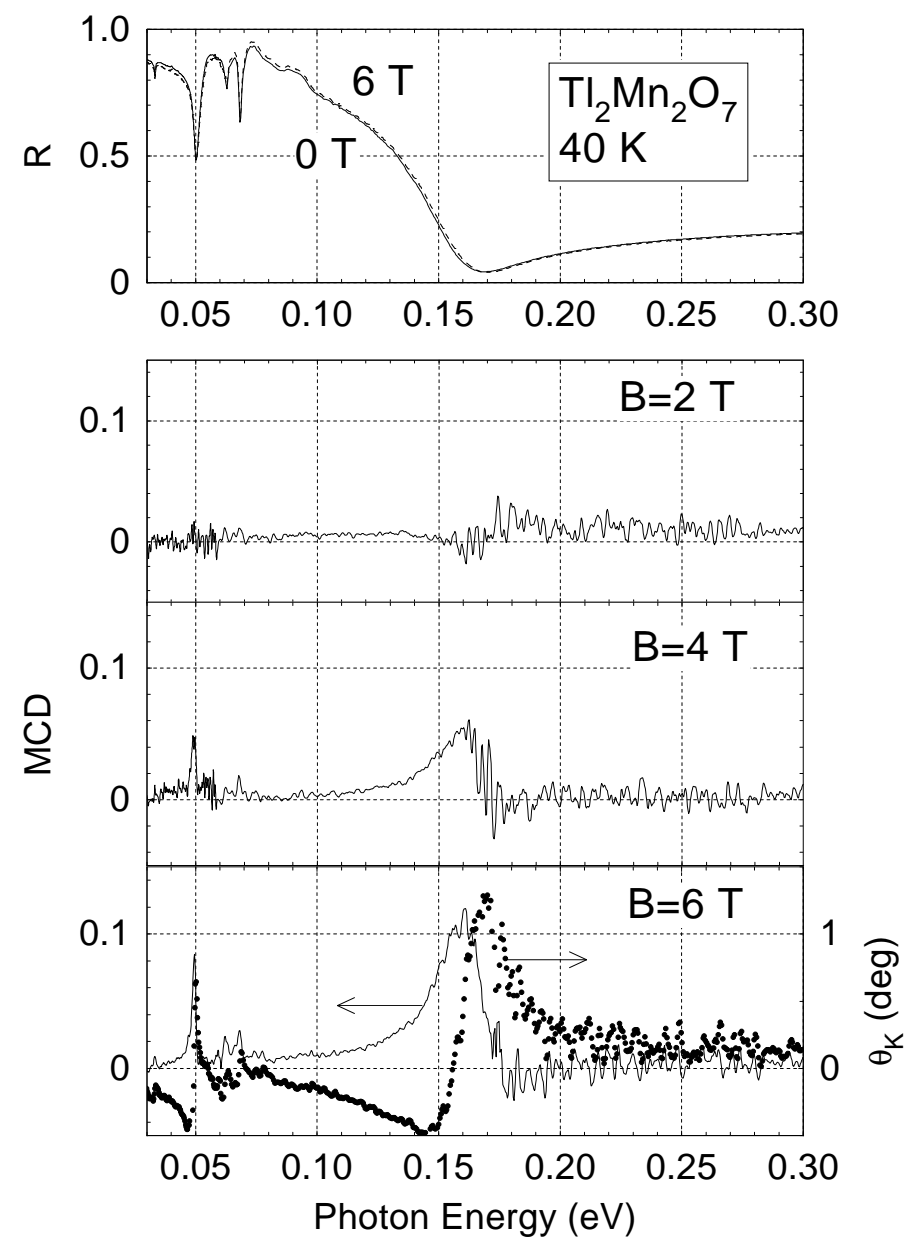

FIG.1

Okamura et al. 


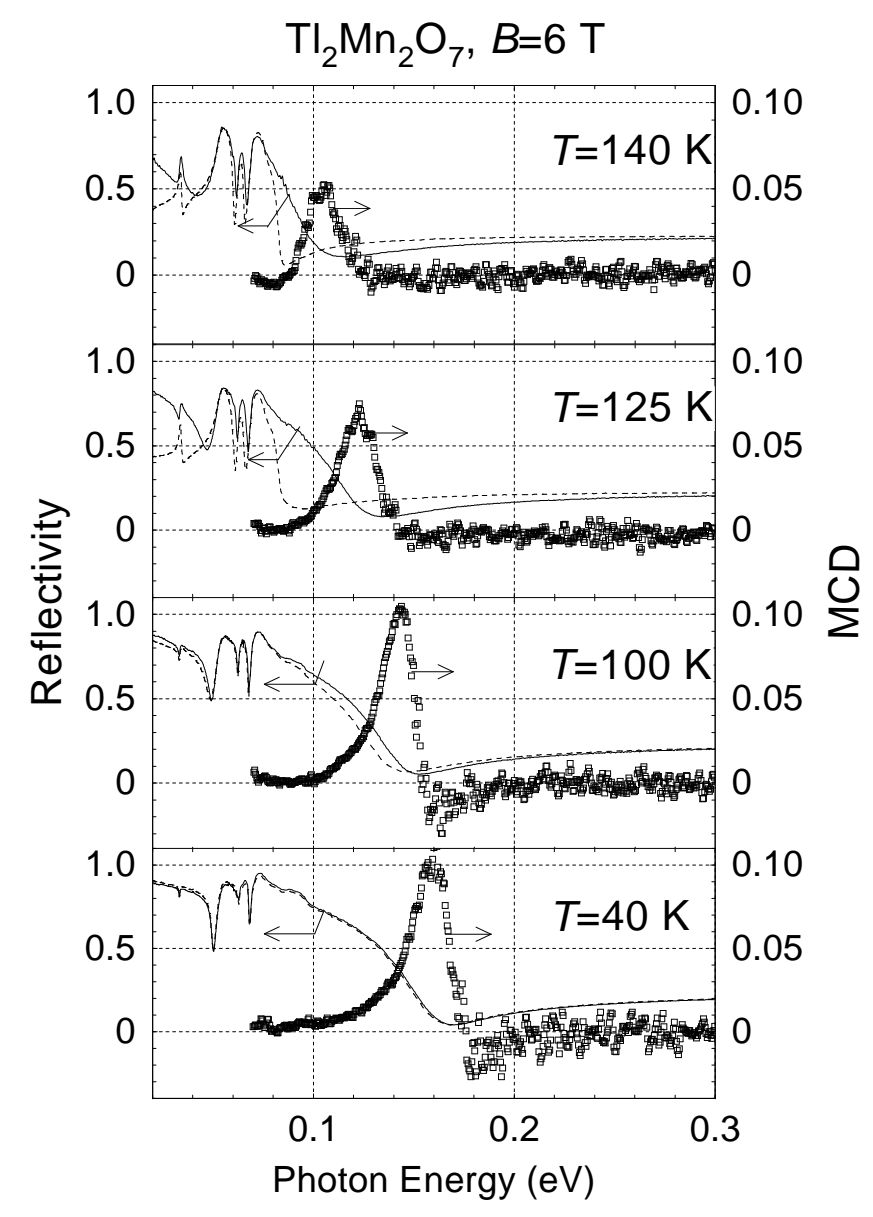

FIG.2

Okamura et al. 

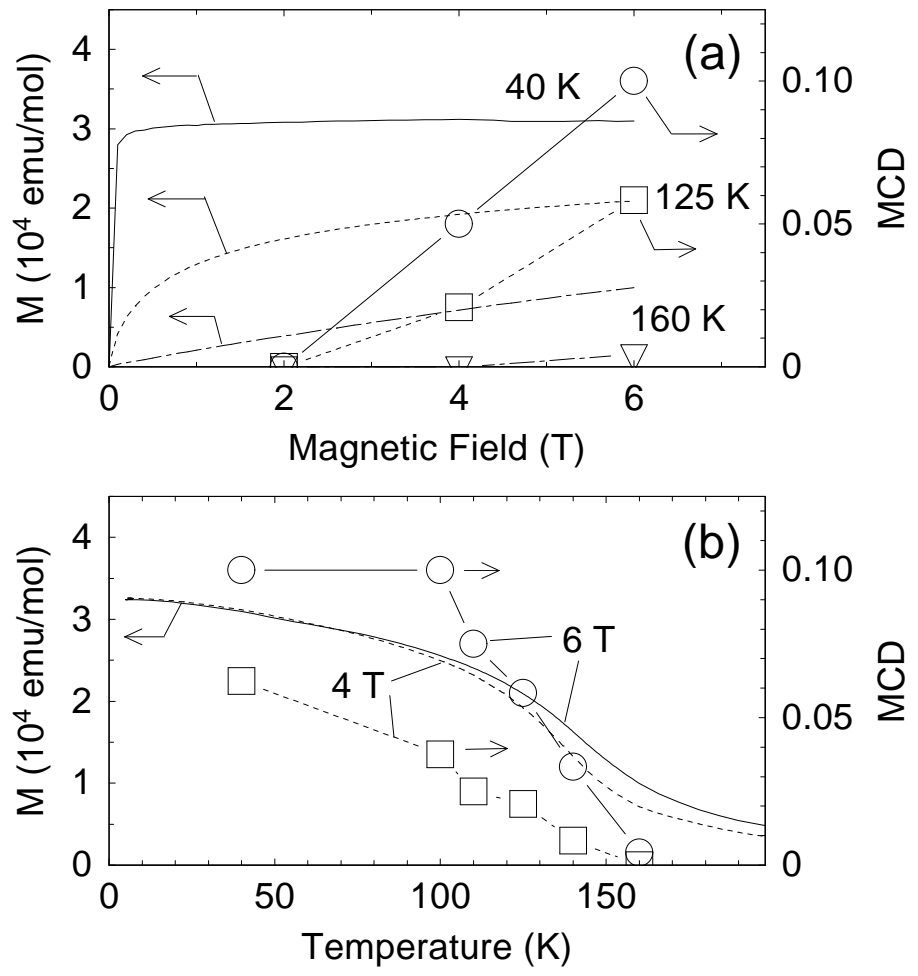

FIG.3 Okamura et al. 


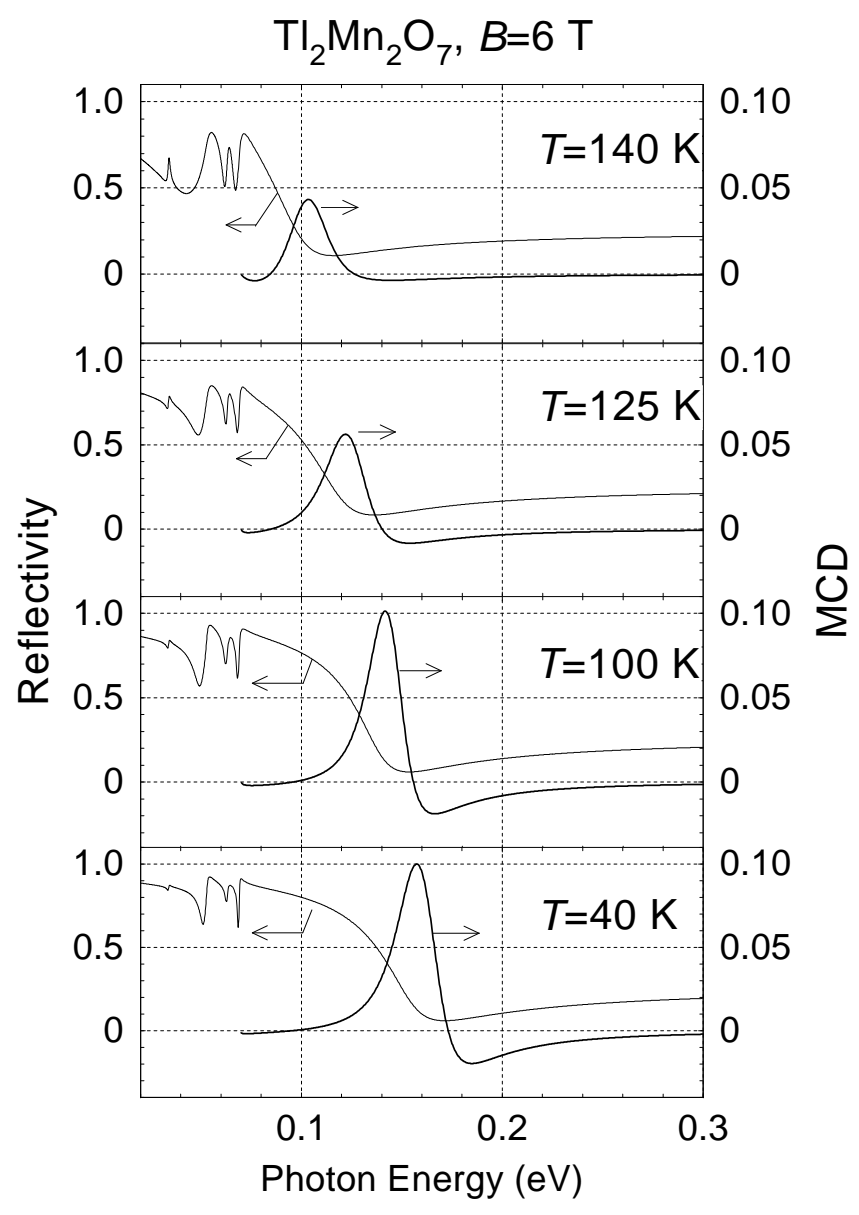

FIG. 4

Okamura et al. 


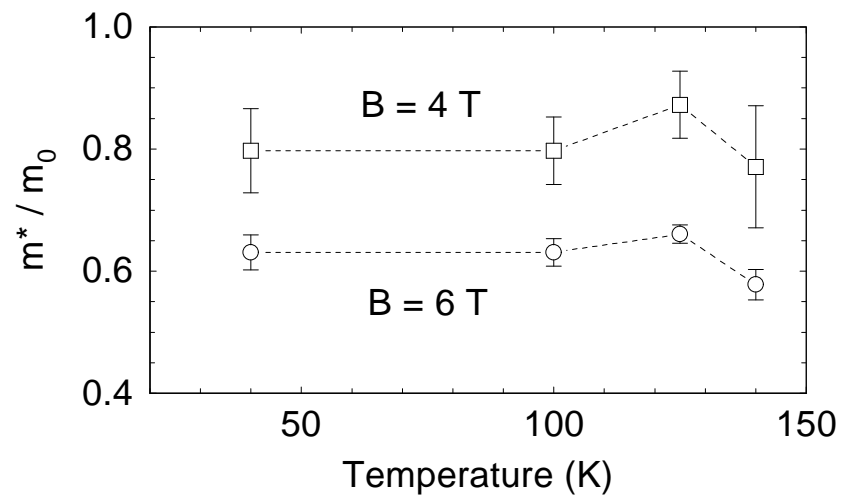

FIG.5

Okamura et al. 